\title{
Percepção de funcionários em uma instituição pública acerca da gestão de resíduos sólidos e dos seus riscos à saúde humana
}

\author{
Perception of employees in a public institution about solid waste management and its \\ risks to human health
}

\section{Percepción de funcionarios en una institución pública acerca de la gestión de residuos sólidos y sus riesgos para la salud humana}

\author{
Giulia Roriz dos Santos ${ }^{1}$ \\ Júlia Tolentino ${ }^{2}$ \\ Marcos $\mathrm{Mol}^{3}$
}

\begin{abstract}
Resumo
O gerenciamento incorreto dos resíduos de serviços de saúde (RSS) apresenta potencial para gerar impactos negativos à saúde humana e ao meio ambiente. Práticas corretas de segregação aliadas à educação ambiental podem reduzir estes danos. Diante disso, o presente estudo teve como objetivo analisar a percepção dos servidores de uma instituição pública de ciência, saúde e tecnologia sobre o gerenciamento dos RSS e seus impactos à saúde. Participaram da pesquisa 101 funcionários dessa instituição, através da aplicação de questionários. Observou-se o nível de conhecimento em relação à classificação dos RSS, ao descarte e à geração de resíduos, e se as etapas do gerenciamento afetam a saúde humana devido ao contato físico com esses resíduos. Foi constatada a existência de significativo conhecimento sobre o tema, mas ainda há a necessidade de continuação dos programas de conscientização e desenvolvimento de práticas adequadas ao gerenciamento dos RSS.
\end{abstract}

Palavras-chave: Percepção. Resíduos sólidos. Resíduos de serviços de saúde. Saúde. Instituição pública.

\begin{abstract}
The incorrect management of healthcare waste $(\mathrm{HCW})$ has the potential to generate negative impacts to human health and the environment. Suitable segregation practices and environmental education can reduce these damages. Hence, the present study aimed to analyze the perception of the employees working in a public institution focused on science, technology and health about the HCW management and its impacts on public health. In this research, 101 employees participated through the questionnaires application. It was observed the knowledge level regarding the classification of HCW and its generation, and whether these management steps can affect human health through physical contact with wastes. The results show the existence of significant knowledge on the subject, but there is still a need to continue awareness programs and develop appropriate practices for the HCW management.
\end{abstract}

Keywords: Perception. Solid waste. Healthcare waste. Health. Public institution.

\section{Resumen}

La gestión incorrecta de los residuos de servicios de salud (RSS) presenta potencial para generar impactos negativos para la salud humana y el medio ambiente. Las prácticas correctas de segregación y educación ambiental pueden reducir estos daños. Por ello, el presente estudio tuvo como objetivo analizar la percepción de los servidores de una institución pública de ciencia, salud y tecnología sobre la gestión de los RSS y sus impactos en la salud.

\footnotetext{
${ }^{1}$ Graduanda em Engenharia Ambiental e Sanitária pelo CEFET-MG e bolsista de Iniciação Científica da Diretoria de Pesquisa e Desenvolvimento da Fundação da Ezequiel Dias (FUNED). Rua Conde Pereira Carneiro, 80. Gameleira. Belo Horizonte, MG. CEP 30510-010. giulia.santos@ funed.mg.gov.br.

${ }^{2}$ Graduanda em Engenharia Ambiental e Sanitária pelo CEFET-MG e bolsista de Iniciação Científica da Diretoria de Pesquisa e Desenvolvimento da Fundação da Ezequiel Dias (FUNED). Rua Conde Pereira Carneiro, 80. Gameleira. Belo Horizonte, MG. CEP 30510-010. julia.tolentino@ funed.mg.gov.br.

${ }^{3}$ Doutor em Saneamento e Meio Ambiente pela UFMG e pesquisador da Diretoria de Pesquisa e Desenvolvimento da Fundação da Ezequiel Dias (FUNED). Rua Conde Pereira Carneiro, 80. Gameleira. Belo Horizonte, MG. CEP 30510-010. marcos.mol@ funed.mg.gov.br.
} 
Participaron de la encuesta 101 funcionarios de esa institución, a través de la aplicación de cuestionarios. Se observó el nivel del conocimiento en relación a la clasificación de los RSS, al descarte y la generación de residuos y si las etapas de la gestión afectan la salud humana, a través del contacto físico con estos residuos en el lugar de trabajo. Podemos notar la existencia de un conocimiento sobre el tema, pero todavía hay la necesidad de continuar los programas de concientización y desarrollo de prácticas adecuadas para la gestión de RSS.

Palabras-clave: Percepción. Residuos sólidos. Residuos de servicios de salud. Salud. Institución pública.

\section{Introdução}

A existência de uma relação entre a má gestão dos resíduos sólidos e a saúde pública é evidente quando analisada a recorrência de enfermidades causadas na história da espécie humana (BARROS, 2012). Segundo Siqueira e Morais (2009), os resíduos sólidos representam um componente indireto na transmissão de doenças, visto que proporcionam condições adequadas para a proliferação de vetores. O cocobacilo Yersinia pestis, por exemplo, é o causador da peste bubônica e se encontra, principalmente, em pulgas. A propagação dessa doença, que devastou grande parte da Eurásia em meados do século XIV, se deu por conta da invasão de uma espécie de rato preto por meio de rotas comerciais, que continha pulgas carregando o microrganismo patogênico (ZIETZ; DUNKELBERG, 2004). Os hábitos domésticos desse vetor, somados com a ausência de hábitos de higiene e de políticas de saúde à época, foram imprescindíveis para a disseminação da doença.

Outras doenças como a febre tifoide, gastroenterites, leptospirose e as hepatites A e B também estão associadas com a gestão inadequada de resíduos sólidos. Os vetores mais comuns são ratos, baratas, moscas e mosquitos, os quais encontram alimentação e abrigo nos resíduos sólidos (LAZZARI; REIS, 2011). Como descrito por Pereira, Lucena e Fernandes (2010), o manejo adequado dos resíduos sólidos é uma característica determinante para a qualidade de vida da sociedade e para a manutenção da qualidade ambiental e da saúde dos profissionais que lidam com esses resíduos.

Para melhor entender a relação entre saúde e resíduos sólidos, é importante ter clareza sobre a definição de resíduos sólidos e seu potencial de representar riscos à saúde humana. De acordo com a norma brasileira NBR 10004/04, da Associação Brasileira de Normas Técnicas - ABNT, resíduos sólidos são produtos da ação de atividades domésticas, do comércio, da indústria, de serviços agrícolas e também de serviços hospitalares. Dentre estes resíduos, há aqueles gerados em serviços de saúde (RSS) que podem apresentar características que potencializam ainda mais os riscos à saúde se comparados aos resíduos domiciliares, quando mal gerenciados. Assim, uma das formas de assegurar um bom gerenciamento na manipulação dos RSS é conhecer sua classificação para, assim, proporcionar uma segregação adequada desses resíduos (ABNT, 2004).

Conforme a Resolução da Diretoria Colegiada (RDC) $n^{\circ}$ 222/18, da Agência Nacional de Vigilância Sanitária - ANVISA, os RSS são gerados em empreendimentos prestadores de serviços à saúde humana e animal, inclusive as unidades móveis de atendimento à saúde, tais como as clínicas médicas e odontológicas, posto de saúde e hospitais. Os RSS são classificados como: grupo A - resíduos biológicos, subdivididos em A1 a A5, conforme o tipo de resíduo; grupo B - resíduos químicos; grupo C - rejeitos radioativos; grupo D - resíduos comuns; e grupo E - perfurocortantes.

As Resoluções RDC n. ${ }^{\circ}$ 222/18 da ANVISA e Conama 358/05 determinam que o manejo dos RSS é de responsabilidade de seus geradores. De acordo com Ramos et al. (2009), o ambiente hospitalar possui a capacidade de promover a saúde, mas também de criar situações de risco para os indivíduos ou agrupamentos humanos, que podem se manifestar com agravos, doenças, lesões, traumas e mortes, quando ocorrem falhas no modo de operação previsto. 
Quando é considerado o contexto do gerenciamento dos RSS, uma das ferramentas adotadas para promover o maior controle dos ambientes geradores é o Plano de Gerenciamento de Resíduos de Serviços de Saúde (PGRSS). Este, de acordo com Barros (2012), é o documento técnico que estabelece diretrizes para o manejo adequado dos RSS, contendo as etapas de segregação, acondicionamento, coleta, armazenamento, transporte, tratamento e disposição final, com a finalidade de reduzir ou eliminar a geração de resíduos e promover o encaminhamento seguro, assegurando a proteção dos trabalhadores e a preservação da saúde pública e do meio ambiente. Dessa forma, o PGRSS deve considerar ações de envolvimento coletivo, e o planejamento do programa deve ser elaborado incluindo todos os setores do estabelecimento e todos os funcionários que nele atuam, definindo-se obrigações e responsabilidades de cada um em relação aos potenciais riscos identificados nas atividades realizadas.

De acordo com Moreschi et al. (2014), a participação ativa dos funcionários da saúde no cumprimento das diretrizes propostas pelo PGRSS é um engajamento essencial, tendo em vista a relação sistêmica entre saúde e ambiente como fator significativo na educação ambiental. Não obstante, verifica-se a necessidade de despertar uma consciência coletiva dos profissionais que atuam nesses ambientes, para se alcançar o gerenciamento efetivo desses resíduos.

Diante disso, nota-se a importância de que todos os indivíduos geradores de RSS estejam cientes dos procedimentos e riscos associados ao manuseio e tratamento dos resíduos. Para isso, é importante avaliar a eficácia das práticas de conscientização adotadas. Segundo Minayo e Sanches (1993), o uso de questionários e entrevistas permite verificar esse conhecimento, uma vez que tal metodologia trabalha com valores, representações, atitudes, crenças, hábitos e opiniões, de forma a adequar-se à complexidade de fatos particulares de cada grupo.

Considerando a importância de verificar a efetividade das capacitações, Pereira (2011), em sua pesquisa sobre a percepção dos profissionais das Unidades de Saúde em estudo, verificou que os participantes demonstraram possuir preocupações em relação ao meio ambiente, pois ressaltaram ter cautela ao gerenciar os RSS para evitar prejuízos ambientais. Em estudo realizado em um hospital de Belo Horizonte/MG, Chagas et al. (2019) identificaram uma busca contínua dos gestores participantes por informações em relação ao descarte correto dos resíduos para promover adequada gestão dos RSS gerados no estabelecimento. Por outro lado, Aragão (2019) demonstrou, através da análise da percepção de funcionários de um hospital do Estado da Bahia, que há um distanciamento entre os geradores de RSS e o processo de gerenciamento, sendo nítido o não aprofundamento ou preocupação dos participantes com o fluxo seguido pelo resíduo até o destino final. Outro estudo, que analisou a percepção ambiental dos moradores de uma cidade acerca dos problemas ambientais causados pelos resíduos e pelo seu gerenciamento incorreto, obteve como resultado um consenso entre os participantes em relação aos prejuízos causados pelo descarte inadequado do lixo, como fator impactante à poluição (FIGUEIREDO, 2013).

Portanto, o presente trabalho teve como objetivo avaliar a percepção de funcionários de uma instituição de ciência, saúde e tecnologia de Minas Gerais acerca do gerenciamento dos resíduos de serviços de saúde e seus impactos à saúde.

\section{Metodologia}

A percepção sobre o processo de gerenciamento dos RSS gerados e sua relação com a saúde foi investigada por meio de uma pesquisa quantitativa, adotando-se a aplicação de questionários individuais. Os funcionários responderam ao questionário durante os meses de novembro a dezembro de 2018. O questionário foi aplicado nas formas online e presencial, para atingir o maior número de pessoas e garantir o acesso aos funcionários que não dispunham de computador durante a rotina de trabalho. 
Ao todo, a pesquisa foi estruturada em dezoito perguntas, todas de múltipla escolha, sobre as características de geração e gerenciamento dos resíduos sólidos e sua relação com a saúde, por existir possibilidade de contato físico no local de trabalho. Além disso, verificou-se possíveis relações das respostas com o vínculo empregatício dos participantes, bem como se os funcionários consideram que a instituição realiza os procedimentos corretos para o gerenciamento dos resíduos. O questionário online foi divulgado via site institucional da empresa e o presencial foi impresso em papel e aplicado na saída do restaurante, no horário do almoço.

No Quadro 1 são apresentadas as principais perguntas contidas no questionário, bem como as respectivas variáveis a elas associadas, que foram, posteriormente, utilizadas nos modelos estatísticos.

\begin{tabular}{|l|l|}
\hline \multicolumn{1}{|c|}{ Descrição da variável } & \multicolumn{1}{c|}{ Pergunta associada } \\
\hline Conhecimento_resíduos & $\begin{array}{l}\text { Como você classifica o seu conhecimento sobre os } \\
\text { riscos à saúde devido ao contato com os resíduos } \\
\text { da instituição estudada? }\end{array}$ \\
\hline Doença_contato & $\begin{array}{l}\text { Você acha que é possível adquirir doenças através } \\
\text { do contato físico com os resíduos sólidos? }\end{array}$ \\
\hline Importância_coleta & $\begin{array}{l}\text { Quão importante você acha que é a coleta de resíduos } \\
\text { da instituição estudada? }\end{array}$ \\
\hline Importância_classificação_resíduos & $\begin{array}{l}\text { Qual a importância para você da classificação dos } \\
\text { resíduos de serviço de saúde? (biológico, químico, } \\
\text { comum, perfurocortante, radioativo) }\end{array}$ \\
\hline Importância_separação & $\begin{array}{l}\text { Quão importante você considera a separação } \\
\text { dos resíduos sólidos? }\end{array}$ \\
\hline Satisfação_coleta & Qual é a sua satisfação em relação a coleta de resíduos? \\
\hline Prejuízo_saúde & $\begin{array}{l}\text { Quão prejudicial os resíduos (em geral) podem ser para } \\
\text { a saúde humana? }\end{array}$ \\
\hline
\end{tabular}

Quadro 1 - Descrição das variáveis presentes no questionário e as perguntas associadas

Fonte: Dados da pesquisa

Houve a realização de pré-teste, de modo a verificar se as questões apresentavam clareza, mesmo para aqueles que não possuíam conhecimento prévio sobre o assunto. De acordo com Teijlingen e Hundley (2001), uma das vantagens de conduzir um pré-teste é a possibilidade de indicar pontos onde a pesquisa possui o potencial de falha, ou onde os instrumentos utilizados estão inapropriados. Dessa maneira, foi possível a realização de importantes ajustes, previamente à coleta definitiva dos dados. Foram aplicados sete questionários prévios, e algumas questões foram adaptadas para um maior entendimento dos participantes.

Por fim, as respostas do questionário impresso foram lançadas na mesma planilha em que estavam consolidados os resultados do formulário online. Realizou-se uma compilação dos dados obtidos por meio de gráficos e tabelas, facilitando a análise da percepção dos participantes. A análise estatística foi feita a partir do software R (versão 3.5.3). Inicialmente, a normalidade dos dados foi testada através do teste de Shapiro-Wilk ${ }^{4}$. Foi testada a correlação

\footnotetext{
${ }^{4} \mathrm{O}$ objetivo desse teste é fornecer uma estatística para avaliar se uma amostra tem distribuição normal. O teste pode ser utilizado para amostras de qualquer tamanho (SHAPIRO; FRANCIA, 1972).
} 
entre as variáveis, adotando-se o Coeficiente de Correlação de Pearson ${ }^{5}$. Outra análise de correlação adotada foi a Principal Component Analysis (PCA), em português, Análise de Componentes Principais ${ }^{6}$, através do pacote FactoMineR. Para todos os testes estatísticos, foi mantido o nível de confiança de 95\%. Além disso, utilizou-se o software Excel 2016 para a interpretação dos dados e elaboração de gráficos e tabelas dinâmicas.

\section{Resultados e discussão}

A importância dos treinamentos no contexto do gerenciamento dos RSS no Brasil tem amparo legal através do Art. 91 da RDC $\mathrm{N}^{\circ} 222 / 18$ da ANVISA, que estabelece a obrigatoriedade das empresas em garantir a existência de um programa contínuo de educação para os trabalhadores e todos aqueles envolvidos nas atividades de gerenciamento de resíduos. Esse programa deve incluir, entre outros temas, a prática de segregação, identificação, classificação e o risco no manejo dos RSS, além de noções básicas sobre o controle de infecções e contaminações químicas. Além disso, no PGRSS da empresa estudada neste artigo, está prevista a prática de ações preventivas sobre saúde ocupacional e segurança do trabalhador, por meio de campanhas educativas sobre os riscos existentes no local de trabalho. Portanto, é esperado que os funcionários possuam algum conhecimento acerca dos riscos relacionados com 0 gerenciamento de RSS.

A instituição pesquisada possui 1020 funcionários. O questionário foi aplicado a $101(10 \%)$ participantes, assim distribuídos: 68 servidores, quinze bolsistas de iniciação científica júnior (BIC Jr.), catorze bolsistas/estagiários, dois terceirizados, um pesquisador convidado e um funcionário de recrutamento amplo. Os dados gerados foram analisados de acordo com o vínculo empregatício dos participantes, uma vez que cada grupo estudado apresentou um conhecimento prévio do assunto estudado. Foram propostos três grupos: servidores e funcionários de recrutamento amplo (maior tempo de vínculo com a instituição); bolsistas, estagiários, terceirizados e pesquisadores convidados (maior rotatividade e menor tempo de vínculo); e BIC Jr. (estudantes do ensino médio com baixo tempo de vínculo com a instituição, sem experiência profissional e com menor grau de instrução formal).

Ao analisar apenas as respostas dos integrantes do programa BIC Jr., que são formados por alunos do Ensino Médio de escolas públicas, 20\% acreditaram que a gripe tinha relação com o contato direto com os resíduos. Na percepção de alunos do Ensino Fundamental II, conforme estudo realizado por Souza e Santos (2016), sobre a relação do meio ambiente com a saúde, $62 \%$ dos participantes alegaram ter contraído gripe em decorrência de transformações ambientais. Os participantes também acreditaram que os seres humanos causam alterações ao meio ambiente local, e destacaram que o problema mais recorrente dessa relação está associado aos resíduos sólidos (77\%).

Ao comparar os resultados dos estudos mencionados, é possível supor que, na instituição alvo da atual pesquisa, o número de participantes que associou gripe com resíduos seja menor devido à oportunidade de esses jovens (BIC Jr.) trabalharem com RSS e, portanto, provavelmente possuírem maior conhecimento acerca do gerenciamento destes. Apesar de poucos trabalhos científicos associarem a maior ocorrência de gripe ao contato com resíduos, em uma pesquisa realizada por Rodrigues et al. (1997) foi constatada a sobrevivência dos vírus da influenza, pólio tipo I, vírus entéricos, hepatites A e B nos resíduos de serviços saúde.

Portanto, percebe-se que há a possibilidade de sobrevivência do vírus da gripe na massa dos RSS e, além da comprovação microbiológica, precisamos levar em consideração outros fatores

\footnotetext{
${ }^{5} \mathrm{O}$ coeficiente de correlação de Pearson (r), também chamado de correlação linear ou r de Pearson, é um grau de relação entre duas variáveis quantitativas e exprime o grau de correlação através de valores situados entre -1 e 1 (MUKAKA, 2012).

${ }^{6}$ Ferramenta de análise multivariada (LYRA et al., 2010).
} 
como via de transmissão adequada, porta de entrada e hospedeiro em estado de suscetibilidade, para que o ser humano possa adquirir a doença (SILVA et al., 2002). Outros estudos também identificaram microrganismos patogênicos presentes nos RSS como Escherichia coli, Pseudomonas aeruginosa, Clostridium sp., Enterococos, Staphylococcus aureus, Mycobacterium tuberculosis, hepatites A e B (MOREL; FILHO, 1997; SILVA et al., 2002). A presença do agente patogênico em si não garante a ocorrência de doenças, porém trata-se de um dos fatores que podem aumentar as chances de ocorrência.

Sobre a pergunta que enfoca a relação entre doenças e o contato direto com resíduos sólidos, as respostas consolidadas estão apresentadas na Figura 1.

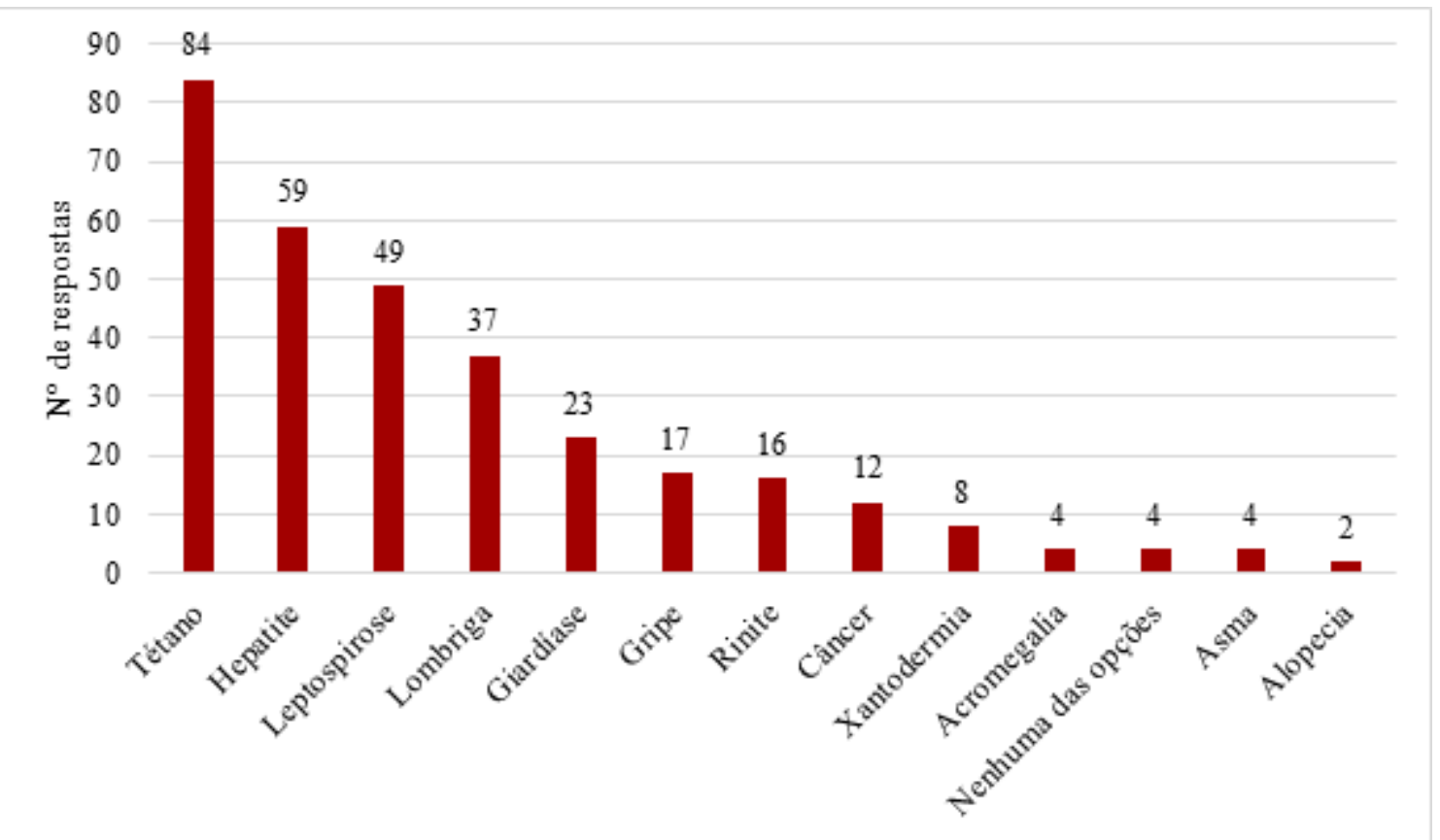

Figura 1 - Frequência de respostas acerca da relação entre doenças e o contato direto com resíduos sólidos Fonte: Dados da pesquisa

Como observado na Figura 1, a maioria dos participantes relacionou tétano e hepatite B com o contato direto com os RSS. Pode-se supor que os funcionários possuem conhecimento prévio sobre a necessidade de prevenção contra essas doenças devido às campanhas de vacinação, previstas antes do início das atividades laborais. De acordo com a NR $\mathrm{n}^{\circ} 32$ do Ministério do Trabalho e Emprego - MTE (BRASIL, 2011), a todos os trabalhadores de serviços de saúde devem ser oferecidas, de forma preventiva, imunizações contra o tétano e hepatite B, além de ministradas instruções sobre os riscos por falta ou recusa de vacinação. Portanto, ao analisar o PGRSS da instituição estudada, foi constatada a existência de programas e treinamentos direcionados para a prevenção de doenças e proteção do meio ambiente.

Outra pergunta, apresentada no questionário, indagava se os participantes do estudo trabalhavam com RSS na rotina de trabalho, e as respostas foram separadas com relação aos tipos de vínculos com a instituição, sendo: $60 \%$ vinculados ao programa Bic Júnior; $41 \%$ bolsistas/estagiários; $45 \%$ servidores/recrutamento amplo.

Quando requisitados a classificar, de zero a dez, o conhecimento acerca dos riscos à saúde causados pelo contato físico com resíduos, a maioria dos participantes (81\%) atribuiu nota superior a seis, conforme a Figura 2. Ou seja, a maioria considerou ter um conhecimento de mediano a ótimo sobre os riscos existentes. Ao analisar essas mesmas respostas, de acordo com o vínculo empregatício, foram encontradas: $94 \%$ dentre servidores/recrutamento amplo, $46 \%$ 
dentre Bic Jr e 65\% dentre bolsistas/estagiários. Dentre os funcionários que trabalham com RSS, $79 \%$ consideraram essa nota superior a sete.

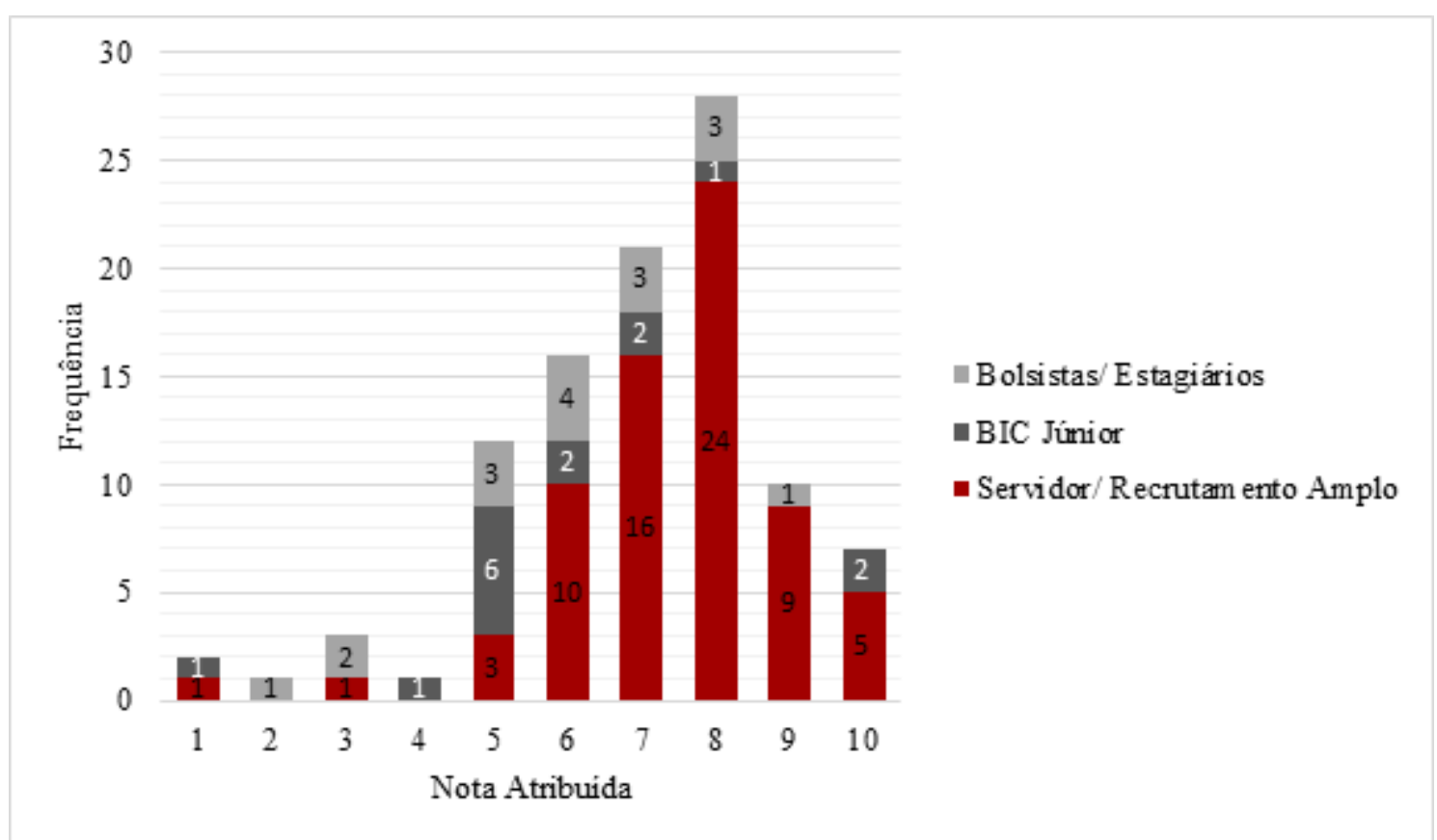

Figura 2 - Classificação do conhecimento sobre os riscos à saúde devido ao contato com os resíduos Fonte: Dados da pesquisa

Como os resultados em relação ao conhecimento pessoal dos participantes acerca dos riscos dos RSS apresentaram valores positivos, é esperado que haja correlação dessa informação com as demais variáveis estudadas neste trabalho, uma vez que um maior conhecimento implica em um maior cuidado no manuseio e, consequentemente, uma maior definição de importância dada às práticas de gerenciamento.

Ao serem questionados sobre quais os tipos de resíduos são mais gerados dentro da instituição, $35 \%$ dos participantes acreditaram que são os resíduos de papel, conforme indicado na Figura 3. Dos funcionários que declararam trabalhar com RSS, $26,7 \%$ disseram que os resíduos orgânicos são os mais gerados, enquanto 48,9\% dos que não trabalham com RSS associaram o resíduo de papel como o mais gerado. Em uma pesquisa realizada na mesma instituição sobre a quantificação dos resíduos gerados, a geração dos resíduos infectantes representou $28,9 \%$ do total de resíduos gerados no período estudado, enquanto os resíduos químicos representaram 17,5\% e os resíduos comuns 49,9\%, nos quais os resíduos de papel e orgânicos estão incluídos (BARBOSA; MOL, 2018). Uma das possíveis razões para a elevada quantidade de respostas sobre os resíduos de papel, classificado como o mais gerado, pode estar associada ao grande número de setores administrativos existentes na empresa que, devido à atividade do trabalho, apresentam normalmente grande geração desse tipo de resíduo. 


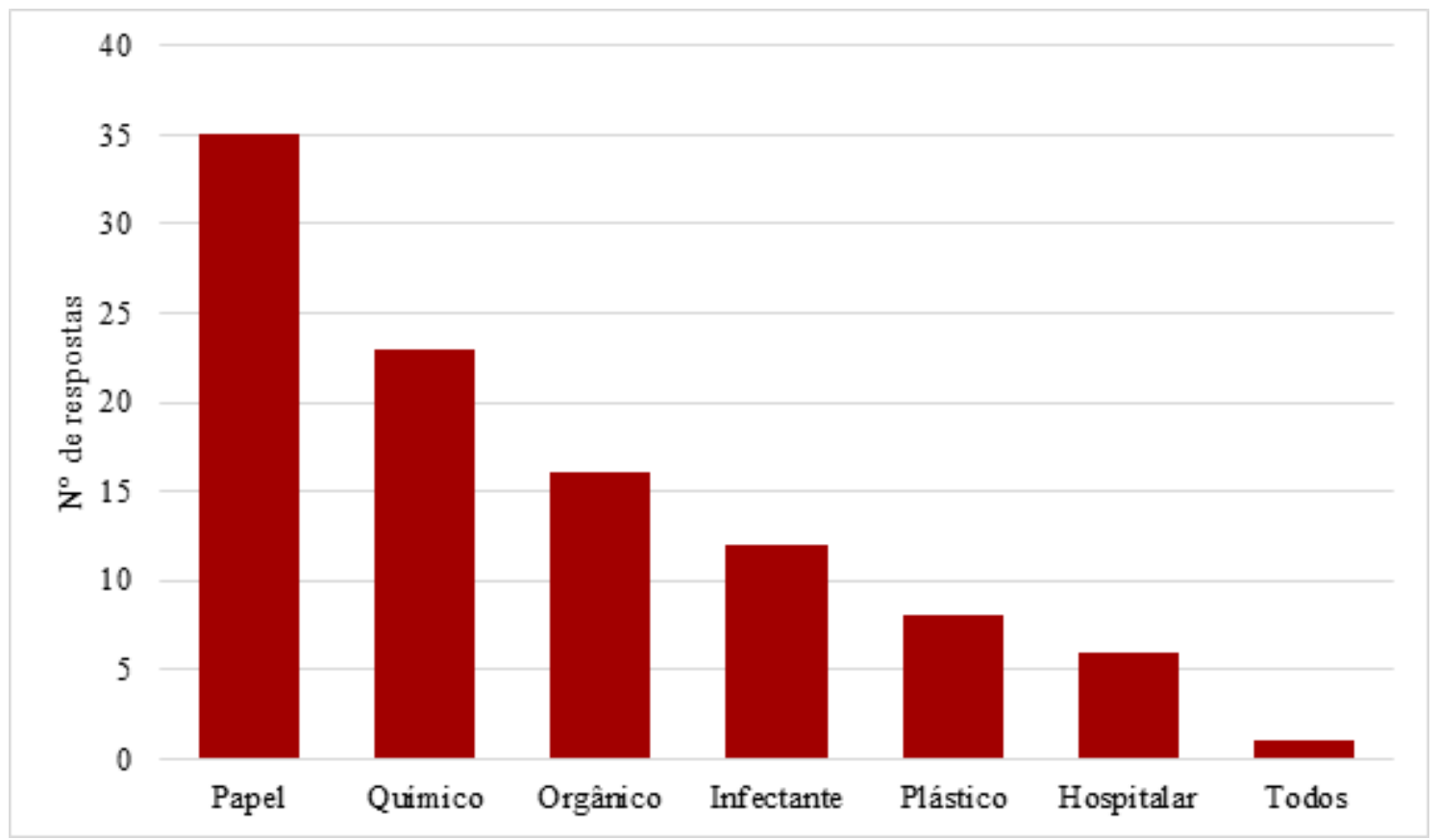

Figura 3 - Frequência de respostas acerca dos tipos de resíduos mais gerados dentro da instituição Fonte: Dados da pesquisa

Para avaliar se a segregação de resíduos em um estabelecimento está de acordo com as normas, o uso de indicadores é um dos possíveis métodos para a verificação da adequação quanto à segregação, uma vez que compara registros reais e históricos da geração de resíduos ao longo do tempo (BELO HORIZONTE, 2011).

Ao analisar estudos similares, reportando a geração de resíduos de papel, os valores de consumo encontrados foram: Instituto Federal de Minas Gerais, campus Governador Valadares, com média de cinquenta folhas de papel por estudante/mês; Empresa de Assistência Técnica e Extensão Rural do Estado de Minas Gerais (EMATER) e Fundação de Amparo à Pesquisa do Estado de Minas Gerais (FAPEMIG), os dados de consumo foram de 188 e 109 folhas de papel A4 por pessoa em um mês, respectivamente (PENNA et al., 2014). De acordo com Almeida et al. (2017), esse cenário pode ser explicado pela maioria das atividades desenvolvidas em instituição públicas que, devido à burocracia existente, exige emissão de relatórios impressos e demais demandas associadas ao consumo desse tipo de material.

Quando avaliada a qualidade da segregação dos resíduos, um ponto fundamental para uma boa prática é o conhecimento da sua classificação e do seu perigo. Tal conhecimento é fundamental para a atuação das pessoas envolvidas no processo de geração, uma vez que a separação deve ser realizada no momento e no local em que ocorre a geração dos resíduos sólidos. Assim, se a pessoa não tem o conhecimento sobre a importância e possibilidade de descartar de forma diferenciada algum tipo de resíduo, utilizando, para isso, lixeiras distintas de acordo com a identificação e característica dos resíduos, consequentemente, não será possível efetivar a segregação no momento da geração. Por isso, o uso de indicadores de segregação é tão importante para demonstrar como a separação está ocorrendo na prática. O treinamento contínuo, incluindo todos os funcionários de uma empresa, é imprescindível para a efetivação de uma segregação eficiente.

Silva (2011) diz que as adequadas separação e destinação dos resíduos nas lixeiras poderão colaborar com a redução do volume dos resíduos sólidos hospitalares, e por consequência, minimizar o risco patológico à sociedade. Assim, a realização de ações durante as atividades laborais é essencial para demonstrar o envolvimento e conscientização com a proteção ambiental. 
Quanto à importância da separação dos resíduos, a nota atribuída predominantemente pelos participantes foi superior a sete. Ao analisar os escores dez atribuídos por classe de vínculo, 94\% dos servidores/recrutamento amplo selecionaram essa resposta, enquanto 93\% dentre os BIC Jr. e $76 \%$ dentre bolsistas/estagiários. Portanto, isso demonstra que grande parte dos funcionários considera relevante a segregação. De maneira análoga, no estudo de Moreschi et al. (2014), os participantes (docentes e egressos) demonstraram, também, uma preocupação voltada à segregação adequada dos RSS, ressaltando que esses resíduos devem ser gerenciados com cautela, uma vez que podem causar prejuízos ao meio ambiente e à qualidade de vida da sociedade. Já os discentes destacaram a importância dos destinos diferenciados e variedade dos resíduos produzidos em centros de saúde. A maior parte dos participantes, em todas as categorias de vínculos empregatícios, atribuiu nota dez quanto à importância de existir uma classificação dos RSS.

\subsection{Análise estatística das respostas}

De acordo com o teste de Shapiro-Wilk realizado com os dados analisados neste estudo, as variáveis foram classificadas como não paramétricos, ou seja, não seguem distribuição normal. Além disso, elaborou-se um modelo de PCA, que foi validado por apresentar componentes principais com dimensões iguais ou acima de $50 \%$, o que explica grande parte da variabilidade dos dados. Entretanto, a análise de PCA para a classe de servidores/recrutamento amplo não atingiu esta meta, indicando que o modelo não possui representatividade para estudar este vínculo.

Ao analisar uma possível correlação entre as respostas dadas quanto à importância da classificação dos resíduos e os prejuízos causados para a saúde humana, foi obtido um valor de $-0,02$, ou seja, não houve correlação entre as variáveis. Essa mesma informação foi comprovada pelo PCA, conforme Figura 4 (superior/esquerda), uma vez que as variáveis apresentaram posição perpendicular entre si. No entanto, quando analisada a mesma associação, restringindose ao tipo de vínculo empregatício, houve uma moderada correlação (valor de 0,43 ) analisando as respostas dos BIC Jr., enquanto para servidores/recrutamento amplo e bolsistas/estagiários, os valores foram de $-0,02$ e $-0,15$ respectivamente, ou seja, sem correlação. Portanto, pode-se constatar a pouca associação entre a importância da classificação dos resíduos com os prejuízos à saúde causados pelos resíduos, contrariando a esperada correlação entre esses tópicos. 

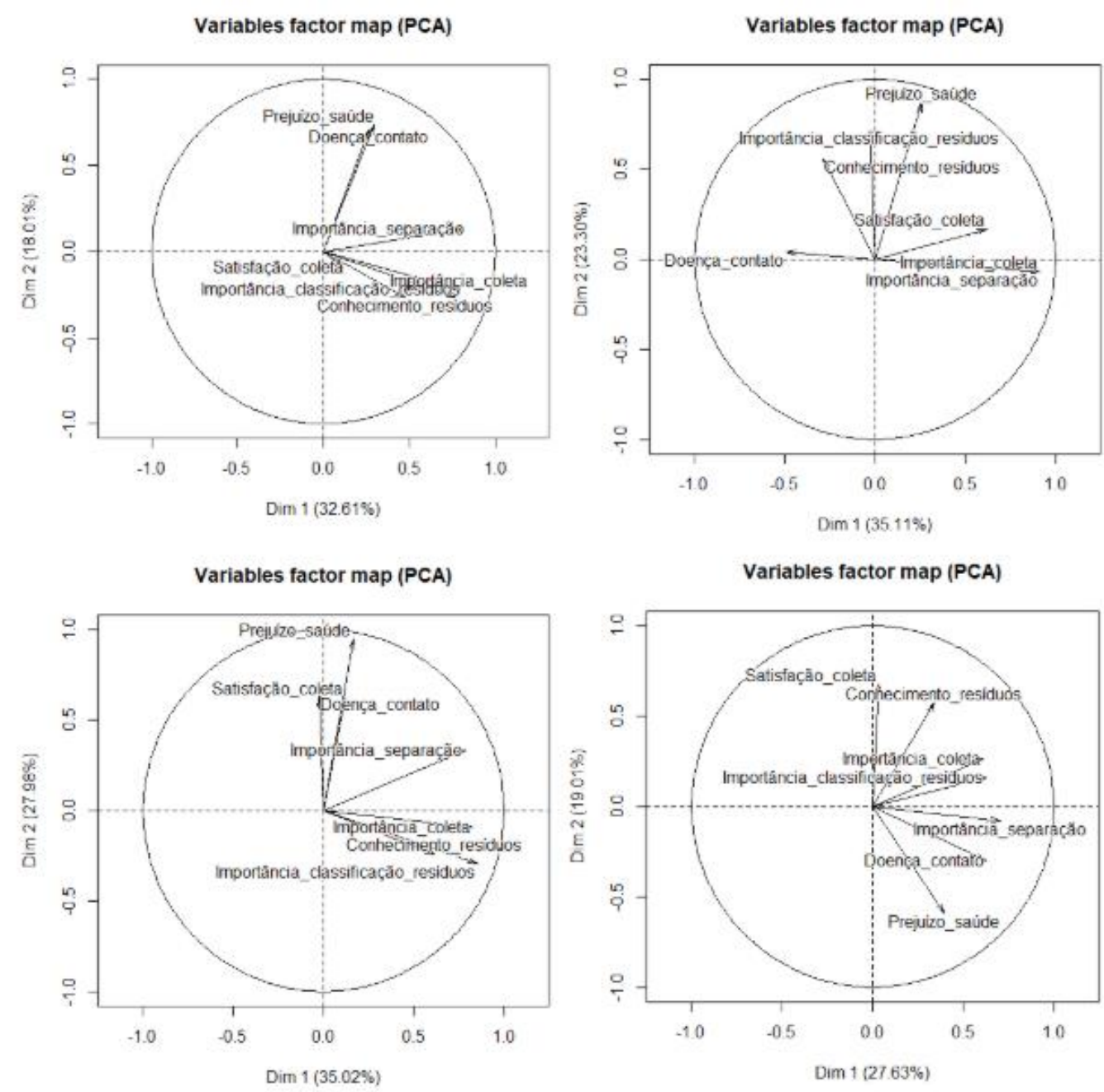
Legenda:
- superior/esquerda: dados das respostas de todos os participantes
- superior/direta: dados das respostas dos bolsistas BIC Jr.
- inferior/esquerda: dados das respostas dos bolsistas/estagiários
- inferior/direita: dados das respostas dos servidores/recrutamento amplo

Figura 4 - Mapa perceptual das variáveis analisadas nos questionários aplicados neste trabalho Fonte: Dados da pesquisa

De forma similar, um estudo realizado por Mendes (2005), em um hospital filantrópico, avaliou a percepção de profissionais da área da enfermagem quanto à classificação dos RSS. O resultado mostrou que os profissionais possuem conhecimentos acerca da classificação, mas não associaram a importância desse conhecimento aos riscos inerentes aos RSS quando mal gerenciados.

Era esperado que houvesse correlação entre as variáveis prejuízo-saúde e doença-contato, uma vez que aqueles que reconhecem que os resíduos apresentam potencial para trazer prejuízos à saúde humana, também deveriam reconhecer que existe a possibilidade de se contrair doenças por meio do contato físico com os RSS. Essa relação é comprovada nas respostas dos participantes, conforme Figura 4 (superior/esquerda). As duas variáveis assumem posição paralela no PCA e se encontram em um mesmo sentido, o que indica uma forte correlação positiva entre elas.

Observou-se, também, que houve correlação entre as variáveis importância-coleta e importância-classificação-resíduos, evidenciada pelo ângulo agudo formado por elas no PCA, 
conforme Figura 4 (superior/esquerda). Isso indica que os participantes que consideraram de grande importância a classificação dos RSS demonstraram tendência a considerar a coleta dos resíduos também como uma importante etapa gerencial. De maneira similar, os participantes da pesquisa realizada por Chagas et al. (2019) apontaram a identificação dos resíduos como ferramenta para viabilizar melhor segregação dos RSS.

Verifica-se que o PCA dos bolsistas/estagiários, conforme Figura 4 (inferior/esquerda), apresenta comportamento semelhante ao PCA de todos os participantes, indicado na Figura 4 (superior/esquerda). Ao analisar a variável satisfação-coleta com as variáveis prejuízo-saúde e doença-contato, foi constatada a existência de uma correlação entre elas. Sendo assim, os bolsistas/estagiários demonstraram relacionar a realização da coleta dos resíduos com o prejuízo a saúde humana e o contato direto com os resíduos. Esse resultado corrobora com o esperado, uma vez que a coleta regular dos resíduos reduz o risco de contato direto com esses materiais.

Observa-se no mapa perceptual dos BIC Jr, ainda na Figura 4 (inferior/direita), que os elementos doença-contato e importância-coleta estão opostos, ou seja, os participantes consideraram que a importância da coleta dos resíduos não tem associação com a chance de se adquirir doenças através do contato físico com os resíduos sólidos. No entanto, esperava-se a existência de uma correlação entre essas variáveis, pois a coleta dos resíduos regularmente garante a integridade do processo de gerenciamento e a falta da coleta pode provocar exposição excessiva e desnecessária dos funcionários aos resíduos sólidos gerados na instituição. Ao verificar as variáveis doença-contato com prejuízo-saúde, ainda considerando apenas as respostas dos BIC Jr., não foi possível identificar correlação entre elas, comprovado pelo ângulo próximo de 90 graus apresentado na Figura 4 (superior/direita). Essa ausência de correlação pode ser explicada pelo pouco tempo dessa classe na instituição e, consequentemente, a pouca experiência com resíduos de serviço de saúde.

A disposição incorreta dos resíduos, tanto hospitalares como domésticos, pode ser foco de propagação de doenças infectocontagiosas, por meio de vetores presentes nesses locais e pela percolação de líquidos gerados, cujo conteúdo pode conter agentes patogênicos. Vale ressaltar que a transmissão de doenças por meio do contato físico com os resíduos sólidos depende de fatores como as condições de susceptibilidade do hospedeiro, a via de transmissão e uma porta de entrada que permita sua inoculação (CHAVES, 2003). O acondicionamento e a disposição final adequada dos resíduos, em especial os RSS, assumem papel de grande importância no que concerne à preservação ambiental e à saúde pública. Diante disso, é importante que exista a percepção acerca da segregação correta e da coleta dos resíduos sólidos, e de que forma essas etapas do gerenciamento de resíduos podem afetar a saúde.

\section{Considerações finais}

Constatou-se, através da análise das percepções dos participantes incluídos nesta pesquisa, que os funcionários atribuem importância à separação dos resíduos sólidos, consideram ter um conhecimento sobre os resíduos e que esses materiais possuem potencial para causar algum dano para saúde. Esse conhecimento era esperado e, portanto, pode-se dizer que as ações de divulgação e capacitação alcançaram êxito quanto aos tópicos elencados. Entretanto, algumas correlações esperadas não foram encontradas, como a percepção acerca da importância da classificação dos resíduos com os prejuízos à saúde, demonstrando que o processo de construção do conhecimento precisa ser debatido de forma constante.

Ao analisar os dados por tipo de vínculo empregatício, percebeu-se uma diferença entre as respostas dadas, possivelmente relacionadas ao grau de conhecimento e treinamento dos participantes. Os servidores, normalmente, apresentam maior tempo de vínculo e uma jornada de trabalho maior dentro da instituição, podendo ter mais chances de terem sido instruídos se 
comparados aos bolsistas/estagiários e BIC Jr. que possuem uma rotatividade maior. Diante disso, podemos sugerir que os treinamentos previstos sobre o PGRSS da instituição estudada, e oferecidos frequentemente na instituição, podem estar produzindo um efeito positivo na disseminação de conhecimento sobre a relação entre resíduos e saúde, analisando sob a ótica dos funcionários da instituição.

Uma alternativa que pode melhorar o gerenciamento dos resíduos de serviços de saúde é a continuidade dos treinamentos aos profissionais, aliado com ações educativas no cotidiano da instituição. Para existir uma cultura de responsabilidade dos funcionários quanto à participação nos procedimentos sobre geração e manuseio de resíduos, é necessária atuação contínua na capacitação dos colaboradores.

\section{Referências}

ALMEIDA, R. et al. Diagnóstico Ambiental de uma Instituição de Ensino Técnico, Integrado e Superior. Revista gestão e sustentabilidade ambiental, Florianópolis, v. 6, n. 3, p. 223-243, Out./Dez. 2017.

AGÊNCIA NACIONAL DE VIGILÂNCIA SANITÁRIA - ANVISA. Resolução $R D C N^{\circ} 222$, de 28 de março de 2018. Regulamenta as Boas Práticas de Gerenciamento dos Resíduos de Serviços de Saúde e dá outras providências. Brasília: Ministério da Saúde/Anvisa, 2018. Disponível em: <http://portal.anvisa.gov.br/documents/10181/3427425/RDC_222_2018_.pdf/c5d3081d-b331-46268448-c9aa426ec410>. Acesso em: 12 Apr. 2019.

ARAGÃO, R. M. B. A. Segregação de resíduos sólidos no bloco operatório de um hospital público estadual: estudo sobre o saber e o fazer dos trabalhadores. 2019. 108 f. Dissertação (Mestrado Profissional em Planejamento Ambiental) - Universidade Católica de Salvador, Salvador, 2019.

ASSOCIAÇÃO BRASILEIRA DE NORMAS TÉCNICAS - ABNT. NBR 10.004: Classificação de resíduos sólidos. Rio de Janeiro: ABNT, 2004.

BARBOSA, F. C. L.; MOL, M. P. G. Proposal of indicators for healthcare waste management: Case of a Brazilian public institution. Waste Management \& Research, Belo Horizonte, v. 36, n. 10, p. $934-$ 941, 2018.

BARROS, R. T. V. Elementos de Gestão de Resíduos Sólidos. Belo Horizonte: Tessitura, 2012. Vol. 1.

BRASIL. Ministério do Trabalho e Emprego. Portaria $n^{\circ}$ 1748, de 30 de agosto de 2011. Aprova a Norma Regulamentadora $n^{\circ} 32$ (Segurança e saúde no trabalho em estabelecimentos de saúde).

Brasília: MTE, 2011. Disponível em: <http://www.normaslegais.com.br/legislacao/portaria-mte-17482011.htm>. Acesso em: 12 apr. 2019.

CHAGAS, C.; SOUSA, C. V.; CUNHA, G. R.; BATISTA, N. K. Gestão de resíduos sólidos: estudo de caso em uma instituição hospitalar em Belo Horizonte - MG. Revista ENIAC Pesquisa, Guarulhos, v.8, n.1, p. 140-153, Jan./Jun. 2019.

CHAVES, L. C. Manipulação de resíduos sólidos de serviços de saúde pela equipe de enfermagem Recomendações. Fundação ABC, São Paulo, v. 28, n. 1, p. 14-18, 2003.

CONSELHO NACIONAL DO MEIO AMBIENTE - CONAMA. Resolução $n^{\circ}$ 358, de 29 de abril de 2005. Dispõe sobre o tratamento e a disposição final dos resíduos dos serviços de saúde e dá outras providências. Brasília: MMA/CONAMA, 2005. Disponível em: <http://www2.mma.gov.br/port/conama/legiabre.cfm?codlegi=462>. Acesso em: 12 apr. 2019. 
BELO HORIZONTE. Município. Comissão Permanente de Apoio ao Gerenciamento de Resíduos de Serviços de Saúde - COPAGRESS. Manual de Regulamento Orientador para a Construção dos Indicadores de Monitoramento, Avaliação e Controle de Plano de Gerenciamento de Resíduos de Serviços de Saúde. Belo Horizonte: Prefeitura Municipal, 2011.

FIGUEIREDO, E. D. C. Análise da percepção ambiental frente ao gerenciamento de resíduos sólidos do município de Saubara/BA. 2013. 63 f. Monografia (Especialização em Gestão Ambiental) Universidade Tecnológica Federal do Paraná, Medianeira, 2013.

LAZZARI, M. A.; REIS, C. B. Os coletores de lixo urbano no município de Dourados (MS) e sua percepção sobre os riscos biológicos em seu processo de trabalho. Ciência \& Saúde Coletiva, Rio de Janeiro, v. 16, n. 8, p. 3437-3442, Jun./2011.

LYRA, W. da S. et al . Classificação periódica: um exemplo didático para ensinar análise de componentes principais. Quím. Nova, São Paulo, v. 33, n. 7, p. 1594-1597, 2010 . Disponível em: $<$ http://www.scielo.br/scielo.php?script=sci_arttext\&pid=S010040422010000700030\&lng=pt\&nrm=iso>. Acesso em: 09 ago. 2019.

MENDES, A. P. A percepção ambiental dos resíduos de serviço de saúde - RSS da equipe de enfermagem de um hospital filantrópico de Araraquara - SP. Dissertação (Mestrado em Desenvolvimento Regional e Meio Ambiente) - Centro Universitário de Araraquara, Araraquara, 2005.

MINAYO, M. C. S.; SANCHES, O. Quantitativo-Qualitativo: Oposição ou Complementaridade?. Cadernos Saúde Pública, Rio de Janeiro, v. 9, n.3, p. 239-262, Jul/Set. 1993.

MOREL, M. M. O.; FILHO, L. A. B. Resíduos de serviços de saúde. In: RODRIGUES, E.A.C. et. al. (Orgs.). Infecções Hospitalares: prevenção e controle. São Paulo: Savier, 1997. p. 519-534.

MORESCHI, C. et al. A importância dos resíduos de serviços de saúde para docentes, discentes e egressos da área da saúde. Revista gaúcha de enfermagem, Porto alegre, v. 35, n. 2, p. 20-26, Jun. 2014.

MUKAKA, M. M. A guide to appropriate use of correlation coefficient in medical research. Malawi Med. Journal, s/l, v. 24, n.3, p. 69-71, Sept. 2012. Available: <https://www.ncbi.nlm.nih.gov/pmc/articles/PMC3576830/>. Access on: 11 apr. 2019.

PENNA, L. F. R. et al. Diagnóstico do consumo de papel A4: o caso do Instituto Federal Minas Gerais Campus Governador Valadares-MG. In: CONGRESSO BRASILEIRO DE GESTÃO AMBIENTAL, 5., 2014, Belo Horizonte. Anais... Belo Horizonte: Instituto Brasileiro de Estudos Ambientais e Saneamento, 2014. Disponível em: 〈http://www.ibeas.org.br/congresso/Trabalhos2014/I-029.pdf〉. Acesso em: 13 abr. 2019.

PEREIRA, S. S.; LUCENA, L. L.; FERNANDES, A. Resíduos de serviço de saúde em um hospital de Campina Grande/PB: gestão e percepção ambiental. Revista Brasileira de Gestão e Desenvolvimento Regional, Taubaté, v. 6, n. 3, p.256-286, 2010.

PEREIRA, S. S. Gestão de resíduos de serviço de saúde e percepção ambiental: estudos de casos em unidades de saúde de Campina Grande/PB. Hygeia: Rev. Bras. Geog. Méd. e da Saúde, Uberlândia, v. 7, n. 12, p. 106-126, Jun./2011.

RAMOS, Y. S. et al. Vulnerabilidade no manejo dos resíduos de serviços de saúde de João Pessoa (PB, Brasil). Ciência \& saúde coletiva, João pessoa, v. 16, n. 8, p. 3553-3559, Ago./Set. 2009. 
RODRIGUES, E. A. C. et al. Infecções Hospitalares: Prevenção e Controle. São Paulo: Sarvier, 1997.

SHAPIRO S. S.; FRANCIA R. An approximate analysis of variance test for normality. J Am Stat Assoc., s/l, v. 67, n. 337, p. 215-216, 1972. Available:

<https://www.tandfonline.com/doi/abs/10.1080/01621459.1972.10481232>. Access on: 12 apr. 2019.

SILVA, A. C. D. N. et al. Critérios adotados para seleção de indicadores de contaminação ambiental relacionados aos resíduos sólidos de serviços de saúde: uma proposta de avaliação. Cadernos Saúde Pública, Rio de Janeiro, v. 18, n. 5, p. 1401-1409, Set./Out. 2002.

SILVA, N. M. Segregação dos resíduos sólidos hospitalares. 2011. 44 f. Monografia (Especialização em Educação Ambiental) - Universidade Federal de Santa Maria, Centro de Ciências Rurais, Santa Maria, 2011.

SIQUEIRA, M. M.; MORAES, M. S. Saúde coletiva, resíduos sólidos urbanos e os catadores de lixo. Ciência e Saúde Coletiva, Rio de janeiro, v. 14, n.6, p. 2115-2122, Dez. 2009. Disponível em: <http://www.scielo.br/scielo.php?script=sci_arttext\&pid=S1413-

$81232009000600018 \& \operatorname{lng}=$ en\&nrm=iso>. Acesso em: 09 ago. 2019.

SOUZA, P. D. F. B; SANTOS, D. B. Percepção de alunos sobre a relação saúde e meio ambiente. Carpe Diem: Revista Cultural e Científica do UNIFACEX, Natal, v. 14, n. 1, p. 54-63, 2016.

TEIJLINGEN, E. R. V; HUNDLEY, V. The Importance of Pilot Studies. Social Research Update, London, v. 35, n. 53, p. 1-4, Jun. 2001. Available: 〈https://sru.soc.surrey.ac.uk/SRU35.PDF>. Access on : 12 aug. 2019.

ZIETZ, B. P., DUNKELBERG, H. The history of the plague and the research on the causative agent Yersinia pestis. International Journal of Hygiene and Environmental Health, s/l, v. 207, n. 2, p. 165178, Nov. 2004. 